\title{
Influence of intertrial interval duration on the intertrial agreement effect in delayed matching-to-sample with pigeons
}

\author{
DOUGLAS S. GRANT \\ University of Alberta, Edmonton, Alberta, Canada
}

\begin{abstract}
Accuracy on even-numbered trials was assessed as a function of (1) the relation between the sample on the immediately preceding trial and that on the current trial and (2) the length of the intertrial interval (ITI) that intervened between odd- and even-numbered trials. A relatively long interval intervened between pairs of trials in the clustered-dyads procedure, whereas this interval was equal to the ITI in the massed-trials procedure. Both procedures revealed an intertrial agreement effect in that accuracy was higher when the sample on the immediately preceding trial was identical rather than opposite. A decrease in the magnitude of this effect at longer ITIs was apparent only in the clustered-dyads procedure. The insensitivity of the intertrial agreement effect to variations in ITI in the massed-trials procedure may reflect floor effects and the carryover of memory from multiple prior trials that mask the true magnitude of the intertrial agreement effect at short ITIs.
\end{abstract}

The delayed matching-to-sample (DMTS) task has proven useful in the analysis of short-term retention in pigeons. In the simplest instance, trials in DMTS tasks begin with a brief presentation of one of two stimuli as the sample. Termination of the sample is followed by a variable delay of a few seconds, and termination of the delay is followed by presentation of two comparison stimuli for a choice. One comparison corresponds to the sample presented on the current trial, and the other corresponds to the alternate sample. Choice of the matching comparison is reinforced, whereas choice of the nonmatching comparison is not. The stimulus that serves as the sample and the left/right position that displays the matching comparison vary randomly across trials. Successive trials within sessions are separated by an intertrial interval (ITI).

A number of studies have varied ITI duration and examined performance on each trial (after the first) as a function of the relation between the sample on the current trial and that on the immediately preceding trial (Edhouse \& White, 1988a, 1988b; Hogan, Edwards, \& Zentall, 1981; Roberts, 1980; Roitblat \& Scopatz, 1983). Thus, on sessions with a short ITI (e.g., $2 \mathrm{sec}$ ), trials are temporally massed, whereas on sessions with a longer ITI (e.g., $20 \mathrm{sec}$ ), trials are more temporally distributed. Three main findings have emerged from these studies. First, overall accuracy is lower in sessions with short ITIs than in sessions with longer ITIs. Second, accuracy is lower on any particular trial if the immediately preceding trial involved

This research was supported by Grant OGP 0443 from the Natural Sciences and Engineering Research Council of Canada. I thank Ronald Kelly for commenting on an earlier version of this article. Correspondence concerning this article may be directed to D. S. Grant, Department of Psychology, University of Alberta, Edmonton, AB T6G 2E9, Canada (e-mail: douglas.grant@ualberta.ca). the alternate sample-the intertrial agreement effect. Third, and of primary relevance to the present article, the intertrial agreement effect is affected little, if at all, by ITI duration. That is, reversing the roles of the stimuli from trial $n-1$ to trial $n$ reduces accuracy on trial $n$ to an equivalent extent at short and long ITIs. Cohen and Armstrong (1996) tested rats, using a procedure conceptually similar to that employed with pigeons, and obtained similar results.

The failure of the intertrial agreement effect to weaken with longer ITIs has had a major impact on theoretical accounts of interference effects in pigeons. In particular, that finding obviates one potential account of the finding that accuracy is higher in sessions with longer ITIs. That account, specifically, would attribute enhanced accuracy with longer ITIs to a dissipation of between-trials interference at longer ITIs. However, the finding that the magnitude of intertrial agreement effect is not reduced at longer ITIs suggests that two mechanisms contribute to interference effects (Edhouse \& White, 1988b; Roitblat, 1984, 1993). One mechanism involves the general ability to process stimuli, a time-dependent ability that is held to be impaired by short ITIs. The dissipation of the processing deficit at longer ITIs is held to result in higher accuracy in sessions with longer ITIs. The second mechanism involves a time-independent carryover of memory from one trial to the next. If the carryover memory conflicts with the memory on the current trial, it may intrude and produce errors.

In contrast to the studies cited above, Grant (1975, Experiment 4) obtained a robust effect of ITI duration on the intertrial agreement effect. In Grant's procedure, trials within sessions were temporally segregated into conditions. In the interference condition, a pair of trials, Trial 1 and Trial 2 , was presented. The sample on Trial 1 corre- 
sponded to the incorrect comparison on Trial 2. The ITI separating Trial 1 and Trial 2 was 0,20 , or $40 \mathrm{sec}$ in any particular session. In the control condition, no Trial 1 preceded Trial 2 . Conditions were temporally segregated by interpolating a $60-\mathrm{sec}$ interval (the intercondition interval, ICI) after the termination of each Trial 2 . Accuracy on Trial 2 was markedly lower in the interference condition than in the control condition when the ITI in the interference condition was $0 \mathrm{sec}$. When the ITI in the interference condition was 20 or $40 \mathrm{sec}$, accuracy in the control and interference conditions was equivalent.

It is not obvious why the intertrial agreement effect should be sensitive to variation in ITI duration only in Grant's (1975) procedure. Nonetheless, it is important to determine the reliability of Grant's finding, and that was one purpose of the present experiments. A second purpose was to isolate the procedural factor(s) that determines whether the intertrial agreement effect is sensitive to variations in ITI. Perhaps the most obvious difference between the procedure employed in Grant's experiments and that employed in other experiments is Grant's use of an ICI that temporally segregated conditions. In the present experiments, ITI was varied in procedures that either did or did not involve such temporal segregation.

The general procedure employed in the experiments reported in this article is illustrated in Table 1. All the sessions were composed of trial dyads; consisting of Trial 1 and Trial 2. In some dyads, the identical dyads, the sample on Trial 2 was identical to that on Trial 1 , and in other dyads, the opposite dyads, the sample on Trial 2 was opposite to that on Trial 1. An ITI, which was varied between sessions, separated Trial 1 and Trial 2 within each dyad. An interdyad interval (IDI) separated successive dyads within a session.

Table 1

Illustration of the Procedure Used in the Experiments Reported in this Article

\begin{tabular}{|c|c|c|c|}
\hline Dyad Type & Events & Dyad Type & Events \\
\hline Opposite & $\begin{array}{l}\text { Trial } 1(\mathrm{R}: \mathrm{R}+/ \mathrm{G}-) \\
\text { ITI } \\
\text { Trial } 2(\mathrm{G}: \mathrm{G}+/ \mathrm{R}-) \\
\text { IDI }\end{array}$ & Identical & $\begin{array}{l}\text { Trial } 1(\mathrm{R}: \mathrm{R}+/ \mathrm{G}-) \\
\text { ITI } \\
\text { Trial } 2(\mathrm{R}: \mathrm{R}+/ \mathrm{G}-) \\
\text { IDI }\end{array}$ \\
\hline Identical & $\begin{array}{l}\text { Trial } 1(\mathrm{R}: \mathrm{R}+/ \mathrm{G}-) \\
\text { ITI } \\
\text { Trial } 2(\mathrm{R}: \mathrm{R}+/ \mathrm{G}-) \\
\text { IDI }\end{array}$ & Opposite & $\begin{array}{l}\text { Trial 1 (G: } \mathrm{G}+/ \mathrm{R}-\text { ) } \\
\text { ITI } \\
\text { Trial } 2 \text { (R: } \mathrm{R}+/ \mathrm{G}-\text { ) } \\
\text { IDI }\end{array}$ \\
\hline Identical & $\begin{array}{l}\text { Trial } 1(\mathrm{G}: \mathrm{G}+/ \mathrm{R}-) \\
\text { ITI } \\
\text { Trial } 2(\mathrm{G}: \mathrm{G}+/ \mathrm{R}-) \\
\text { IDI }\end{array}$ & Opposite & $\begin{array}{l}\text { Trial } 1(\mathrm{R}: \mathrm{R}+/ \mathrm{G}-\text { ) } \\
\text { ITI } \\
\text { Trial } 2(\mathrm{G}: \mathrm{G}+/ \mathrm{R}-\text { ) } \\
\text { IDI }\end{array}$ \\
\hline Opposite & $\begin{array}{l}\text { Trial } 1(\mathrm{G}: \mathrm{G}+/ \mathrm{R}-) \\
\text { ITI } \\
\text { Trial } 2(\mathrm{R}: \mathrm{R}+/ \mathrm{G}-) \\
\text { IDI }\end{array}$ & Identical & $\begin{array}{l}\text { Trial } 1(\mathrm{G}: \mathrm{G}+/ \mathrm{R}-) \\
\text { ITI } \\
\text { Trial } 2(\mathrm{G}: \mathrm{G}+/ \mathrm{R}-) \\
\text { IDI }\end{array}$ \\
\hline
\end{tabular}

Note-The parenthetical material that follows each trial specifies the trial configuration: sample ( $G$, green; $R$, red) is given prior to the colon, and comparison configuration after the colon. + and - designate correct and incorrect comparisons. ITI, intertrial interval; IDI, interdyad interval.
A procedure similar to that employed by Grant (1975) was implemented by setting the IDI, equivalent to Grant's ICI, at a relatively long duration (i.e., $60 \mathrm{sec}$ ), which was constant across sessions. This procedure will be referred to as the clustered-dyads procedure. A procedure similar to that employed in other studies of interference (cited earlier in this article) was implemented by setting the IDI to the same value as the ITI. This procedure will be referred to as the massed-trials procedure.

In all the experiments reported in this article, the delay on Trial 1 was always $0 \mathrm{sec}$. Prior research has shown that the primary source of interference is the comparison stimulus pecked, rather than the sample stimulus presented, on Trial 1 (Edhouse \& White, 1988b; Roberts, 1980; Roitblat \& Scopatz, 1983). Use of a 0-sec delay on Trial 1 resulted in high concordance $(>97 \%$, collapsed across experiments) between the sample presented and the comparison selected on Trial 1 . This ensured that nominally identical and opposite dyads, with rare exceptions (i.e., when an error occurred on Trial 1), were also functionally identical and opposite dyads.

Experiments 1 and 2 involved a between-experiments comparison of the clustered-dyads and massed-trials procedures, and Experiment 3 involved a within-experiment comparison of these procedures. If Grant's (1975) finding is reliable, the clustered-dyads procedure should reveal an intertrial agreement effect that is sensitive to ITI duration. Specifically, the intertrial agreement effect should be stronger at shorter than at longer ITIs. Moreover, if the temporal segregation of dyads is critical to obtaining that result, the intertrial agreement effect should be insensitive to ITI duration in the massed-trials procedure.

\section{EXPERIMENT 1}

The first experiment was similar to that reported by Grant (1975, Experiment 4) and employed the clustereddyads procedure. The IDI that separated termination of Trial 2 and onset of Trial 1 between dyads was fixed at $60 \mathrm{sec}$. The ITI that separated termination of Trial 1 and onset of Trial 2 within dyads was fixed within sessions and varied across the values 0,20 , and $60 \mathrm{sec}$ between sessions. It was anticipated that the results would replicate those reported by Grant in that (1) an intertrial agreement effect would be found (i.e., higher Trial 2 accuracy when Trial 2 was identical to Trial 1 than when it was opposite to Trial 1) and (2) the intertrial agreement effect would be sensitive to ITI duration (i.e., a stronger intertrial agreement effect at shorter than at longer ITIs).

\section{Method}

Subjects. Five Silver King pigeons (Columba livia), each approximately 1.5 years old, were maintained at $80 \%$ of their free-feeding weight. The birds were housed individually in wire-mesh cages and were given unrestricted access to water and health grit. Home cages were kept in a colony room, illuminated on a 12:12-h light:dark cycle, with light onset at $0600 \mathrm{~h}$. Each bird had prior experience in delayed simple discriminations involving line orientation stimuli. 
Apparatus. Training and testing was conducted in five identical operant chambers, each measuring $29.0 \times 29.0 \times 24.0 \mathrm{~cm}$ (height $\times$ length $\times$ width). In each chamber, a horizontal alignment of three circular pecking keys (each $2.5 \mathrm{~cm}$ in diameter, with side keys separated edge-to-edge from the center key by $3.0 \mathrm{~cm}$ ) was centered along one end wall. The key alignment was raised $22.5 \mathrm{~cm}$ from the barred-floor base of the chamber. Affixed behind each key was an Industrial Electronics (Van Nuys, CA) in-line projector. All three projectors were capable of transilluminating two colors, either red or green. A force greater than $0.15 \mathrm{~N}$ applied to any key was recorded as a keypeck. The top edge of a $5.0-\mathrm{cm}$-wide $\times 5.5-\mathrm{cm}$-high rectangular opening was located $9.0 \mathrm{~cm}$ directly beneath the edge of the middle key. This opening provided access to a retractable food magazine. A 28-V lamp, recessed within the magazine opening, was illuminated whenever the hopper was raised. Each chamber was enclosed in a sound- and light-attenuating booth. Within each booth, an exhaust fan provided ventilation and, supplemented by an external white noise generator, contributed masking auditory stimulation. All the experimental booths were isolated in the same darkened running room. Experimental events were controlled from and responses were recorded by a microcomputer located in an adjoining room. Experimental sessions were conducted daily 6-7 days per week and began at approximately the same time each day.

Procedure. The subjects were trained to match red and green sample stimuli to red and green comparison stimuli. Sessions consisted of 64 trials, and each trial began with a 1-sec termination of the overhead houselight as a preparatory signal (the houselight remained illuminated at all other times within a session). The sample, which was presented immediately after the preparatory signal, consisted of a 5 -sec presentation of red or green on the center key. After $5 \mathrm{sec}$, the sample was accompanied by presentation of green on one side key and red on the remaining side key (simultaneous matchingto-sample). A single peck to either comparison stimulus terminated the sample and both comparisons. A peck to the red comparison after a red sample and a peck to the green comparison after a green sample were designated correct and thus resulted in reinforcement, consisting of $3 \mathrm{sec}$ of access to mixed grain from the food hopper. Alternatively, a peck to green after red and a peck to red after green were designated as incorrect matches and thus resulted in nonreinforcement, consisting of $3 \mathrm{sec}$ without access to mixed grain. Within each session for each subject, the sample was randomly determined, with the restriction that, over all trials within a session, the two samples appeared equally often. The side key location of the correct comparison stimulus was varied randomly across trials, with the restriction that, over all the trials with each sample within a session, the correct comparison stimulus appeared equally often on the left and right key. A 10-sec ITI separated each trial within a session.

A total of 80 sessions of training were conducted. A correction procedure, in which an incorrect response resulted in repetition of the trial, was in force during the first 12 sessions of training. Accuracy, collapsed across Sessions 9-12, was 93.5\%. The correction procedure was discontinued for the next 4 sessions (13-16), during which accuracy was $96.3 \%$. In the final 4 sessions of training (17-20), the sample was terminated immediately prior to onset of the comparison stimuli ( 0 -sec DMTS), and accuracy was $94.7 \%$. During the next 60 sessions of training, one of three equiprobable delays intervened between termination of the sample and onset of the comparison stimuli. Across these sessions, the delays were gradually increased in length and were 0,1 , and $3 \mathrm{sec}$ for 12 sessions, 0,2 , and $4 \mathrm{sec}$ for 8 sessions, 0,3 , and $5 \mathrm{sec}$ for 16 sessions, 0,4 , and $7 \mathrm{sec}$ for 12 sessions, and 0,5 , and $10 \mathrm{sec}$ for 12 sessions. Collapsed across the final 4 sessions of training, percent correct was $98.4 \%, 88.0 \%$, and $79.7 \%$ at delays of 0,5 , and $10 \mathrm{sec}$, respectively.

Testing sessions were composed of 24 trial dyads. The initial trial in each dyad (Trial 1) always involved a 0-sec delay. The second trial in each dyad (Trial 2) involved one of three equiprobable delays- 0 , 5 , and 10 sec. An ITI, which was fixed within sessions and varied across sessions, separated termination of Trial 1 (end of reinforcement or nonreinforcement period) and onset of Trial 2 (occurrence of a 1-sec preparatory signal) within each dyad. The ITIs employed were 0,20 , and $60 \mathrm{sec}$. An IDI of $60 \mathrm{sec}$ separated termination of Trial 2 and onset of Trial 1 between each dyad. Note that functional ITI and IDI durations were $4 \mathrm{sec}$ longer (3-sec reinforcement/ nonreinforcement period plus 1 -sec preparatory signal) than the nominal values.

Data were recorded only on Trial 2 within each dyad. Sample color and position of the correct comparison stimulus were balanced within delay on all the Trial $2 \mathrm{~s}$ within a session. On a random 12 dyads within each session, the sample on Trial 2 was identical to that on Trial 1 (identical). On the remaining 12 dyads within each session, the sample on Trial 2 was opposite to that on Trial 1 (opposite). A total of 24 testing sessions were conducted, 8 with each ITI. The order of testing ITIs was random, with the restriction that each ITI was tested once in each set of 3 consecutive sessions.

Statistical analyses in all the experiments reported in this article employed $p<.05$ to define the critical region for rejection of the null hypothesis.

\section{Results and Discussion}

Figure 1 shows retention functions on Trial 2 collapsed across the eight sessions of testing at each ITI. An intertrial agreement effect is apparent in that accuracy on Trial 2 was higher when Trial 2 was preceded by an identical Trial 1 than when it was preceded by an opposite Trial 1. Moreover, the intertrial agreement effect was sensitive to ITI duration in that the intertrial agreement effect was stronger at the 0 -sec ITI than at the longer ITIs of 20 and $60 \mathrm{sec}$. Also, the intertrial agreement effect was stronger at longer delays than at the 0 -sec delay, although this effect could reflect a ceiling artifact because accuracy was close to $100 \%$ at the 0 -sec delay.

A dyad type (identical and opposite) $\times$ delay $\times$ ITI analysis of variance (ANOVA) was performed on the data from Trial 2. The ANOVA revealed significant main effects of dyad type $[F(1,4)=59.25]$ and delay $[F(2,8)=$ $15.43]$. Two interaction terms were also significant. The dyad type $\times$ delay interaction $[F(2,8)=8.90]$ confirms that the intertrial agreement effect was stronger at longer than at shorter delays. Most important, the dyad type $\times$ ITI interaction $[F(2,8)=6.54]$ confirms that accuracy on Trial 2 was more strongly influenced by ITI when the preceding Trial 1 was opposite than when it was identical. In other words, the intertrial agreement effect was attenuated at longer ITIs. No other terms reached statistical significance.

To further analyze the dyad type $\times$ ITI interaction, two sets of simple main effects analyses (Kirk, 1968, pp. 179182) were performed. The first set revealed that accuracy on Trial 2 was affected by ITI duration when the preceding Trial 1 was opposite $[F(2,8)=8.75]$, but not when it was identical $(F<1)$. The second set compared the two dyad types at each ITI and revealed that the intertrial agreement effect was significant at the 0 - and 20 sec ITIs $[F \mathrm{~s}(1,4)=129.93$ and 18.17 , respectively $]$ but was not significant at the $60-\sec \operatorname{ITI}[F(1,4)=4.16]$. 


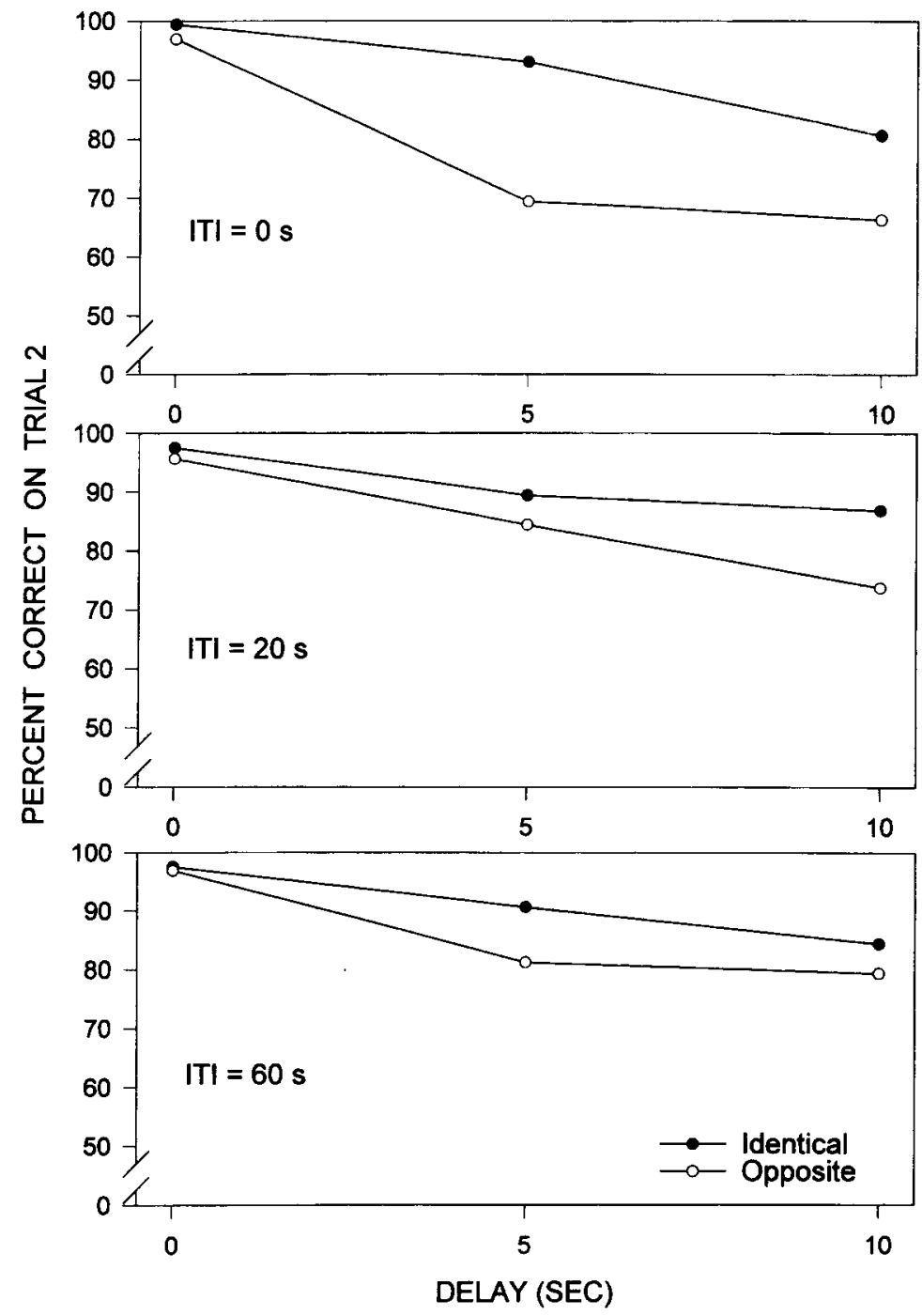

Figure 1. Percentage of correct responses on Trial 2 in identical and opposite dyads as a function of delay in Experiment 1 (clustered-dyads procedure). Each graph plots performance at one of the three intertrial interval (ITI) durations.

The present experiment replicated Grant's (1975, Experiment 4) finding that the intertrial agreement effect is sensitive to ITI duration. In particular, the intertrial agreement effect was strongest at the shortest ITI, weakened progressively as ITI lengthened, and, statistically, was absent at the longest ITI. Moreover, the degree of temporal separation between Trial 1 and Trial 2 affected Trial 2 accuracy only when Trial 1 was opposite to Trial 2 . The second experiment addressed the question of whether use of a fixed and relatively long IDI is necessary to produce this pattern of results.

\section{EXPERIMENT 2}

The second experiment was identical to the first, with one exception. In Experiment 1, the IDI that separated ter- mination of Trial 2 and onset of Trial 1 between dyads was fixed at $60 \mathrm{sec}$. In Experiment 2, the IDI varied between sessions and was equivalent to the ITI duration. Thus, Experiment 2 employed the massed-trials procedure, in contrast to Experiment 1, in which the clustered-dyads procedure was employed. In the present experiment, the ITI and IDI were either 0,20 , or $60 \mathrm{sec}$ in each session. If use of a fixed, relatively long IDI is critical to revealing sensitivity of the intertrial agreement effect to ITI duration, such sensitivity should be absent in the present experiment.

\section{Method}

Subjects and Apparatus. The subjects and apparatus were the same as those in Experiment 1.

Procedure. With one exception, testing was identical to that in Experiment 1 . Specifically, the IDI separating the dyads was set to the same duration as the ITI for that session. 


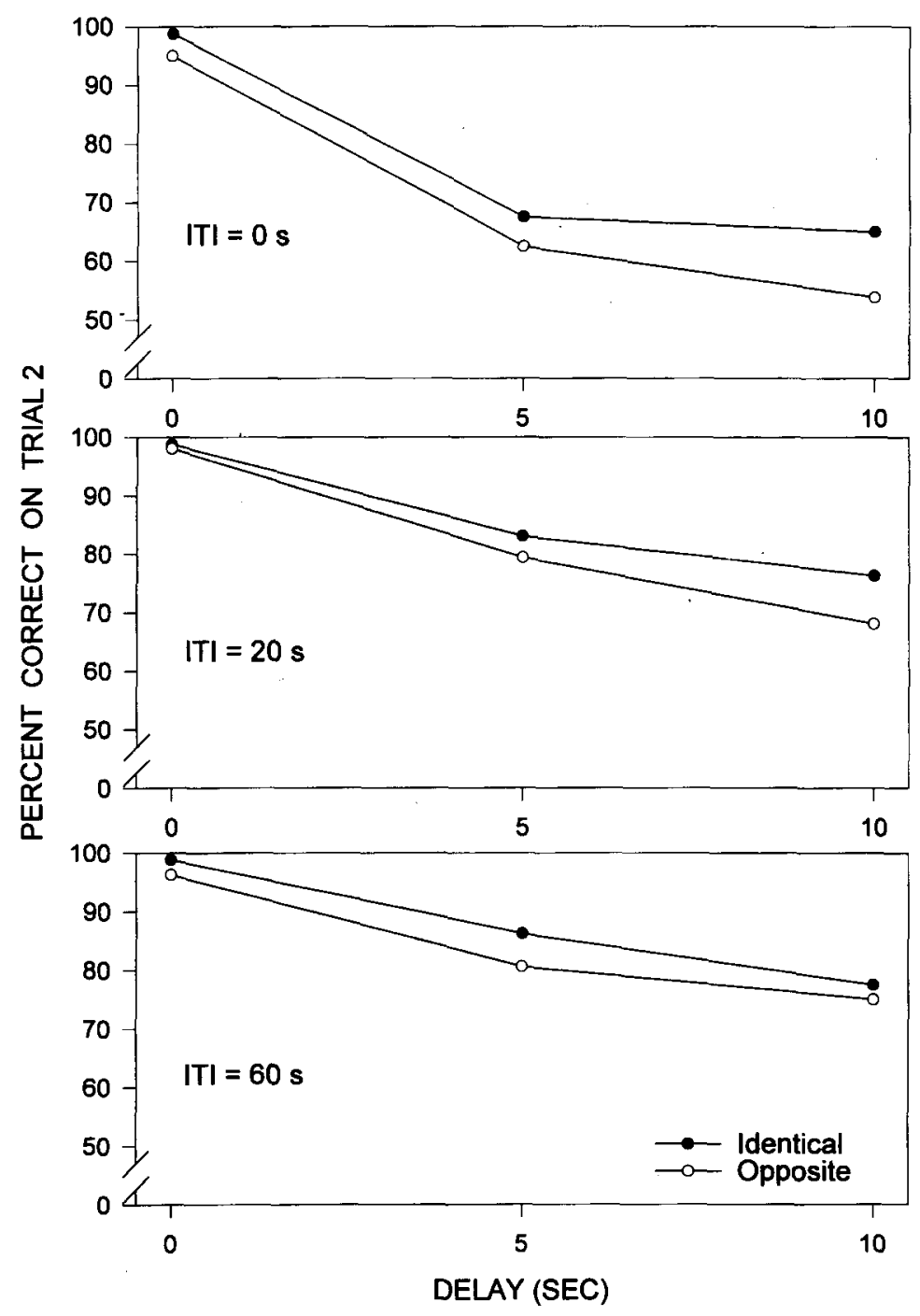

Figure 2. Percentage of correct responses on Trial 2 in identical and opposite dyads as a function of delay in Experiment 2 (massed-trials procedure). Each graph plots performance at one of the three intertrial interval (ITI) durations.

\section{Results and Discussion}

Figure 2 shows retention functions on Trial $2 \mathrm{col}-$ lapsed across the eight sessions of testing at each ITI. As in Experiment 1, an intertrial agreement effect is apparent in that accuracy on Trial 2 was higher when Trial 2 was preceded by an identical Trial 1 than when it was preceded by an opposite Trial 1. Also as in Experiment 1, when the preceding Trial 1 was opposite, accuracy on Trial 2 was facilitated by increases in ITI. Three additional findings shown in Figure 2 are each in contrast to those of Experiment 1 . First, there is little suggestion that the intertrial agreement effect was sensitive to ITI duration. Second, when the preceding Trial 1 was identical, accuracy on Trial 2 was facilitated by increases in ITI. Third, there is little evidence that the intertrial agreement effect was stronger at longer than at shorter delays.
A dyad type (identical and opposite) $\times$ delay $\times$ ITI ANOVA revealed significant main effects of dyad type $[F(1,4)=12.37]$, ITI $[F(2,8)=27.82]$, and delay $[F(2,8)$ $=138.40]$. The significant ITI $\times$ delay interaction $[F(4,16)=6.92]$ reflects the finding that accuracy at longer delays was facilitated to a greater extent by increases in ITI. The fact that, in contrast to Experiment 1, the dyad type $\times$ ITI interaction was not significant $(F<1)$, reveals that accuracy on Trial 2 was enhanced to an equivalent extent by increases in ITI regardless of whether the preceding trial was opposite or identical. No other terms reached statistical significance.

The results of the present experiment are in accord with those of other studies that employed the massedtrials procedure (Edhouse \& White, 1988a, 1988b; Hogan et al., 1981; Roberts, 1980; Roitblat \& Scopatz, 1983) and 
contrast with those obtained with the clustered-dyads procedure (Grant, 1975; Experiment 1 in the present article). In the massed-trials procedure, reductions in ITI produce a general deterioration in accuracy, likely reflecting a deficit in sample processing. Hence, higher accuracy at longer ITIs reflects primarily an alleviation of this processing deficit, resulting in enhanced accuracy regardless of the relation between events on the current trial and those on the immediately preceding trial. In the clustered-dyads procedure, in contrast, higher accuracy at longer ITIs reflects primarily an alleviation of carryover memories from the immediately preceding trial.

\section{EXPERIMENT 3}

The first two experiments confirmed that the massedtrials and clustered-dyads procedures produce interference effects that are different in important ways. Moreover, those experiments also suggested that the critical difference between the procedures is the presence or absence of a relatively long IDI. The present experiment involved a more powerful comparison between the two procedures. Specifically, sessions involving the massed-trials procedure alternated with those involving the clustereddyads procedure. If the present experiment is successful in replicating the results of Experiments 1 and 2, it would provide definitive evidence that (1) the procedures produce intertrial agreement effects that are differentially sensitive to ITI duration and (2) the critical difference between the procedures is the presence or absence of a relatively long IDI.

\section{Method}

Subjects and Apparatus. The subjects and apparatus were the same as those in Experiment 1.

Procedure. Thirty-two sessions of testing were conducted; 16 involved the massed-trials procedure (i.e., IDI = ITI), and 16 involved the clustered-dyads procedure (i.e., IDI $=60 \mathrm{sec}$ ). Within each procedure, 8 sessions involved a 0 -sec ITI, and 8 involved a 60-sec ITI. Note that when sessions involved a 60 -sec ITI, massedtrials sessions and clustered-dyads sessions were identical (i.e., both the ITI and the IDI were $60 \mathrm{sec}$ in both procedures). Massed-trials and clustered-dyads sessions alternated across sessions. Across each set of 4 consecutive sessions, each procedure was tested once with each ITI. All other aspects of the procedure were identical to those in Experiments 1 and 2.

\section{Results and Discussion}

Figure 3 shows retention functions on Trial 2 collapsed across the eight sessions of testing at each ITI in the clustered-dyads procedure. The pattern of results is highly similar to that in Experiment 1. Most important, as in Experiment 1 , the intertrial agreement effect was stronger at the shorter than at the longer ITI. A dyad type $\times$ delay $x$ ITI ANOVA revealed significant main effects of dyad type $[F(1,4)=15.80]$ and delay $[F(2,8)=23.13]$. Two interaction terms were also significant. The dyad type $X$ delay interaction $[F(2,8)=5.45]$ confirms that the intertrial agreement effect was stronger at longer than at shorter delays. The dyad type $\times$ ITI interaction $[F(1,4)=$

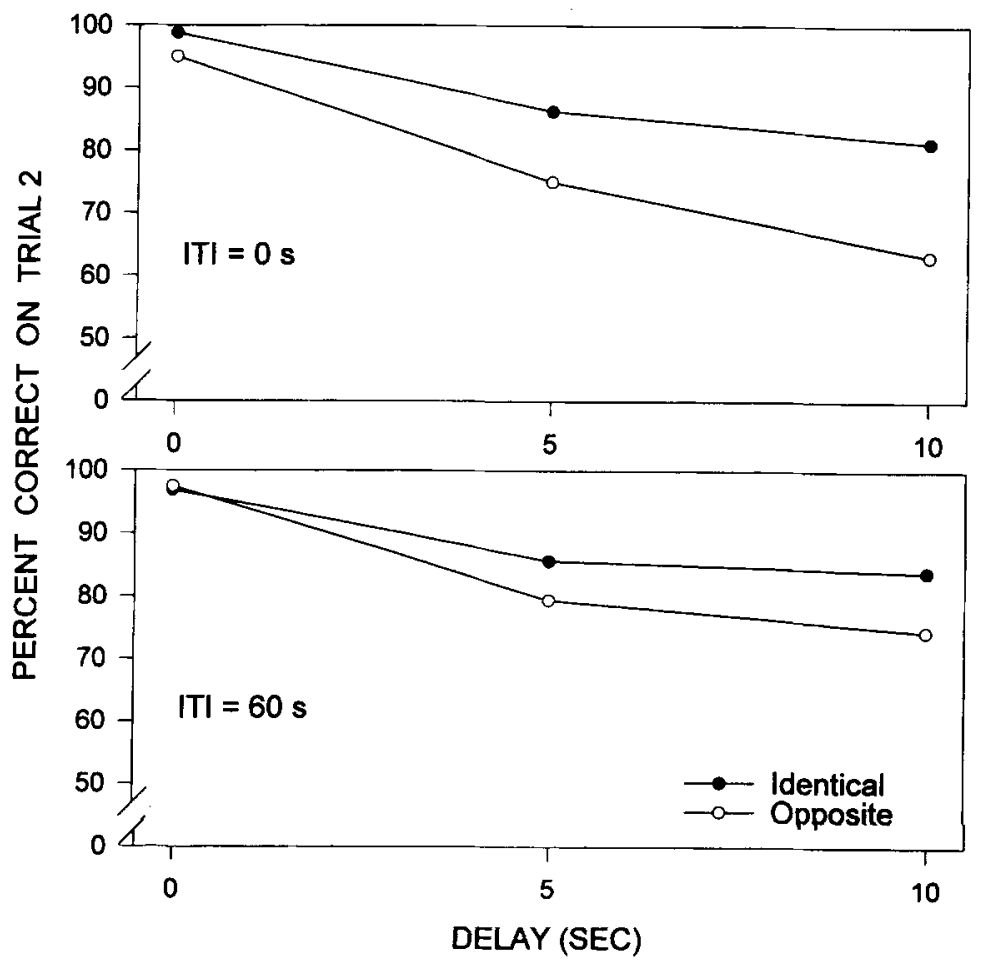

Figure 3. Percentage of correct responses on Trial 2 in identical and opposite dyads as a function of delay in the clustered-dyads procedure in Experiment 3. Each graph plots performance at one of the two intertrial interval (ITI) durations. 
8.21] confirms that accuracy on Trial 2 was more strongly influenced by ITI when the preceding Trial 1 was opposite than when it was identical. In other words, the intertrial agreement effect was attenuated at the longer ITI. No other terms reached statistical significance.

To further analyze the dyad type $\times$ ITI interaction, two sets of simple main effects analyses were performed, as was done in Experiment 1. The first set revealed that accuracy on Trial 2 was affected by ITI duration when the preceding Trial 1 was opposite $[F(1,4)=8.21]$, but not when it was identical $(F<1)$. The second set compared the two dyad types at each ITI and revealed that the intertrial agreement effect was significant at both the 0 - and the 60 -sec ITIs $[F \mathrm{~s}(1,4)=15.13$ and 13.53 , respectively]. On a statistical basis, the present data are identical to those reported in Experiment 1, with one exception: A significant intertrial agreement effect was obtained at the 60 -sec ITI in the present experiment, but not in Experiment 1.

Figure 4 shows retention functions on Trial 2 collapsed across the eight sessions of testing at each ITI in the massed-trials procedure. The pattern of results is highly similar to that in Experiment 2. Most important, as in Experiment 2, the intertrial agreement effect was not influenced by ITI duration. A dyad type $x$ delay $\times$ ITI ANOVA revealed significant main effects of dyad type $[F(1,4)=8.61]$, ITI $[F(1,4)=44.68]$, and delay $[F(2,8)=$ $110.94]$. The significant ITI $\times$ delay interaction $[F(2,8)$ $=8.12]$ reflects the finding that accuracy at longer de- lays was facilitated to a greater extent by increases in ITI. Importantly, as in Experiment 2, the dyad type $\times$ ITI interaction was not significant $(F<1)$, revealing that accuracy on Trial 2 was enhanced to an equivalent extent by increases in ITI regardless of whether the preceding trial was opposite or identical. No other terms reached statistical significance. On a statistical basis, the present data are identical to those reported in Experiment 2.

The results confirm the findings from Experiments 1 and 2. Specifically, when dyads are temporally segregated in the clustered-dyads procedure, (1) the intertrial agreement effect is stronger at shorter than at longer ITIs, and (2) accuracy on Trial 2 is enhanced by increases in ITI only when the immediately preceding Trial 1 is opposite. When dyads are not temporally segregated in the massed-trials procedure, (1) the intertrial agreement effect is not influenced by ITI duration, and (2) accuracy on Trial 2 is enhanced by increases in ITI to an equivalent extent regardless of whether the immediately preceding Trial 1 is identical or opposite.

\section{EXPERIMENT 4}

In the preceding experiments, the intertrial agreement effect was assessed by comparing accuracy on trials preceded by an identical trial with that on trials preceded by an opposite trial, as has typically been the case in studies using the massed-trials procedure (Edhouse \& White, 1988a, 1988b; Roberts, 1980; Roitblat \& Scopatz, 1983).

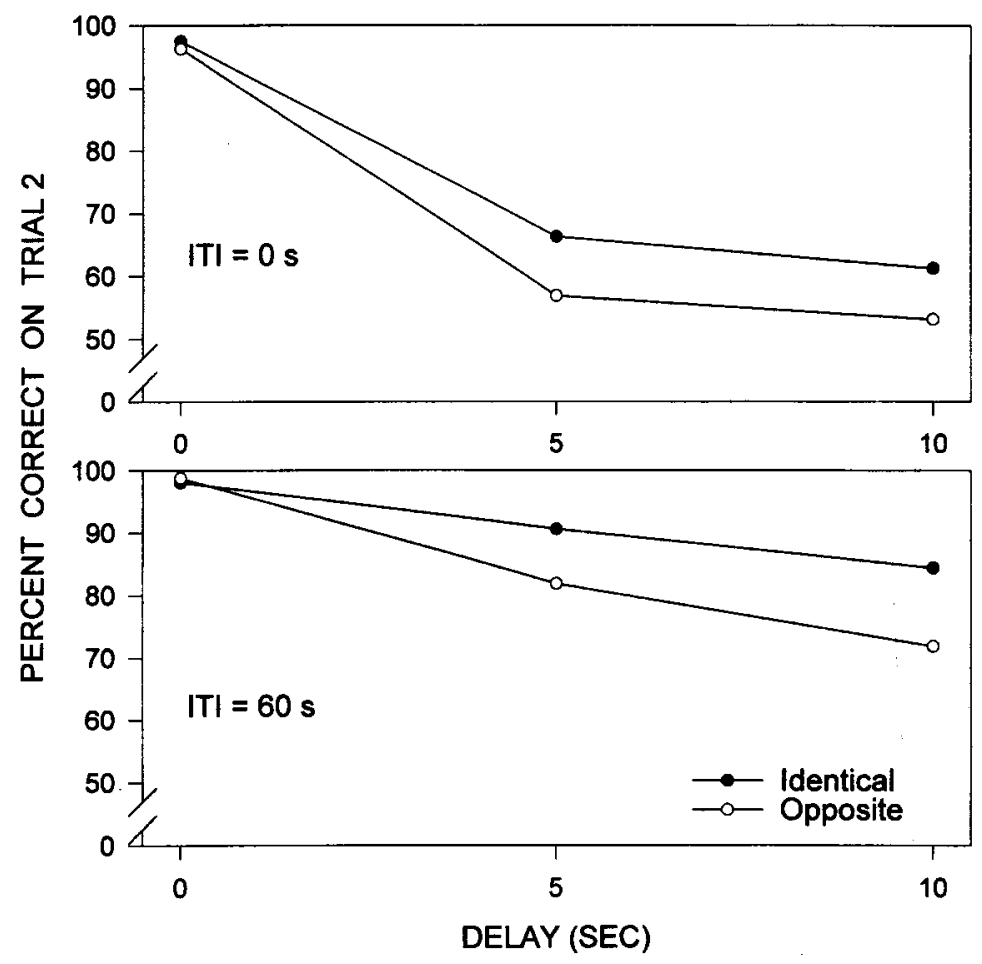

Figure 4. Percentage of correct responses on Trial 2 in identical and opposite dyads as a function of delay in the massed-trials procedure in Experiment 3. Each graph plots performance at one of the two intertrial interval (ITI) durations. 
Such a comparison, however, does not permit an assessment of the extent to which the intertrial agreement effect is a function of (1) proactive facilitation caused by an identical preceding trial and/or (2) proactive interference caused by an opposite preceding trial.

Hogan et al. (1981) demonstrated that the intertrial agreement effect in the massed-trials procedure is exclusively a function of proactive interference caused by an opposite preceding trial. They compared performance on trials as a function of whether the correct and incorrect comparison stimuli on the immediately preceding trial maintained the same role (positive transition), were reversed in roles (negative transition), or were absent (neutral transition). No evidence of proactive facilitation was obtained, in that accuracy was equivalent in positive and neutral transitions. Marked proactive interference was present, however, in that accuracy on negative transitions was lower than that on either positive or neutral transitions.

Grant's (1975) findings revealed that proactive interference also contributes to the intertrial agreement effect in the clustered-dyads procedure. Grant found lower Trial 2 accuracy when Trial 2 was opposite Trial 1 than when either Trial 1 was omitted or the Trial 1 stimuli were absent on Trial 2. Hence, a proactive interference effect was demonstrated. However, Grant did not employ identical dyads, and therefore, it is possible that proactive facilitation may have contributed to the intertrial agreement effect reported in the clustered-dyads procedure in the experiments reported in this article. The present experiment assessed this possibility by comparing accuracy on Trial 2 as a function of whether the preceding Trial 1 was identical, opposite, or not presented.

\section{Method}

Subjects. Eight naive Silver King pigeons ( $C$. livia), each approximately 1 year old, were maintained and housed as specified in Experiment 1.

Apparatus. The apparatus was the same as that in Experiment 1.
Procedure. The subjects were initially trained to eat grain from the raised and illuminated magazine and were then autoshaped to peck red and green when presented on any of the three pecking keys. Following preliminary training, the subjects were trained on identity matching involving red and green sample and comparison stimuli. The procedure during matching training was identical to that specified in Experiment 1 .

Testing sessions were composed of 36 trial dyads, and a 60 -sec IDI followed termination of each Trial 2. On a random 12 dyads within each session, Trial 1 was omitted (none). Hence, none dyads involved only a single trial (Trial 2), separated from termination of the preceding Trial 2 by the IDI. The remaining 24 dyads were equally divided between identical and opposite dyads. Identical and opposite dyads were as specified in Experiment 1. The ITI that separated Trial 1 and Trial 2 on identical and opposite dyads was fixed at $0 \mathrm{sec}$. Twelve testing sessions were conducted. All other aspects of the procedure were as specified in Experiment 1.

\section{Results and Discussion}

Figure 5 shows retention functions on Trial 2 collapsed across the 12 sessions of testing. There was little suggestion of a proactive facilitation effect, in that accuracy on identical and none dyads was equivalent. However, there was a robust proactive interference effect, in that accuracy on opposite dyads was markedly lower than that on identical and none dyads.

A dyad type (none, identical, and opposite) $\times$ delay ANOVA revealed significant main effects of dyad type $[F(2,14)=27.29]$ and delay $[F(2,14)=30.49]$ and a significant dyad type $\times$ delay interaction $[F(4,28)=$ 3.84]. No other terms reached statistical significance. Separate dyad type $x$ delay ANOVAs were performed to compare performance in the three dyad types. These analyses revealed that accuracy on identical and none dyads did not differ, in that neither the main effect of dyad type $[F(1,7)=1.29]$ nor the dyad type $\times$ delay interaction $(F<1)$ was significant. On the other hand, the analyses comparing performance on (1) opposite and identical dyads and (2) opposite and none dyads each revealed significant main effects of dyad type $[F \mathrm{~s}(1,7)=27.34$ and

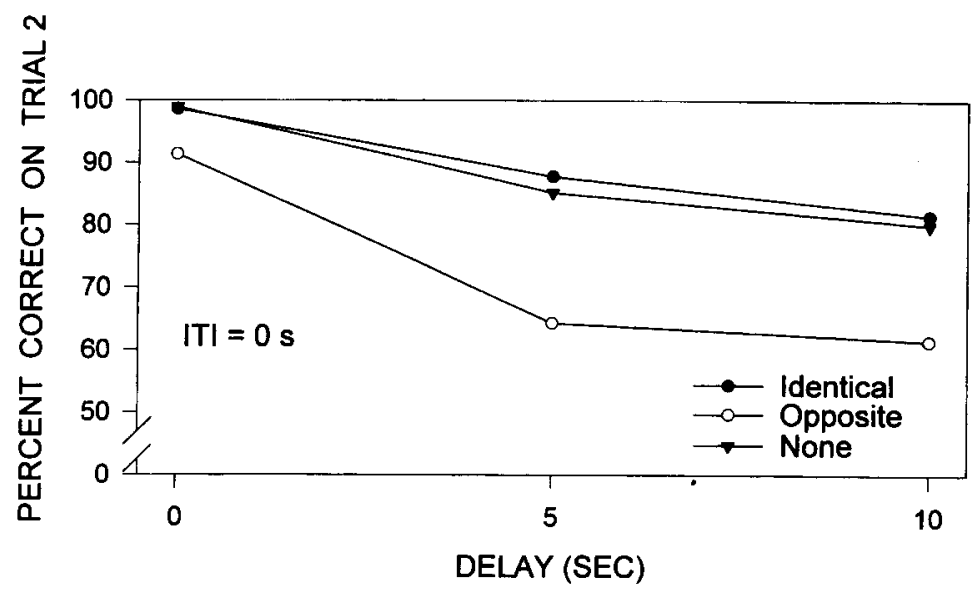

Figure 5. Percentage of correct responses on Trial 2 in none, identical, and opposite dyads as a function of delay in the clustered-dyads procedure in Experiment 4. 
31.70 , respectively] and a significant dyad type $\times$ delay interaction $\left[F_{\mathrm{S}}(2,14)=4.23\right.$ and 3.89 , respectively].

These results replicate Grant's (1975) finding that, in a clustered-dyads procedure, preceding Trial 2 by an opposite Trial 1 results in markedly lower accuracy than when Trial 2 is not preceded by a Trial 1 -a proactive interference effect. Moreover, as in the massed-trials procedure, there is no evidence of a proactive facilitation effect. Specifically, Trial 2 accuracy was unaffected by whether Trial 1 was omitted or was identical to Trial 2. Hence, as in the massed-trials procedure (Hogan et al., 1981), the intertrial agreement effect in the clustereddyads procedure is primarily, and perhaps exclusively, a function of proactive interference generated by a conflicting preceding trial.

\section{GENERAL DISCUSSION}

The present experiments compared performance in two procedures used to investigate proactive interference in pigeon short-term retention. In the clustered-dyads procedure, pairs of DMTS trials are temporally segregated from other pairs by a relatively long (e.g., $60 \mathrm{sec}$ ) interval (the IDI) that remains constant across sessions. Trials within a pair, or dyad, are separated by an ITI that varies in length across sessions. Accuracy on the second trial within each dyad, Trial 2, is assessed on the basis of the delay on Trial 2 and the relation between the samples on Trial 1 and Trial 2, identical or opposite. The second procedure, the massed-trials procedure, is highly similar, except that successive dyads within a session are separated by an ITI equivalent in length to that separating trials within dyads.

Prior research using procedures similar to those just described has revealed an intertrial agreement effect (e.g., Edhouse \& White, 1988a, 1988b; Grant, 1975; Hogan et al., 1981; Roberts, 1980; Roitblat \& Scopatz, 1983), an effect replicated in the experiments reported in this article. The intertrial agreement effect refers to the finding that accuracy on Trial 2 is higher when the immediately preceding Trial 1 involves the same sample than when Trial 1 involves the alternate sample.

The focus in the present experiments was an apparent difference in the sensitivity of the intertrial agreement effect to variations in ITI in the two procedures. In the clustered-dyads procedure, the intertrial agreement effect is reduced at longer ITIs (Grant, 1975, Experiment 4). In contrast, in the massed-trials procedure, the magnitude of the intertrial agreement effect is affected little, if at all, by length of ITI (Edhouse \& White, 1988a, 1988b; Hogan et al., 1981; Roberts, 1980; Roitblat \& Scopatz, 1983). Experiments 1 and 2, using a between-experiments comparison, and Experiment 3, using a within-experiment comparison, confirmed the reliability of the betweenprocedure difference in sensitivity of the intertrial agreement effect to variations in ITI. In particular, the clustereddyads procedure revealed a reduction in the intertrial agreement effect as ITI was increased from 0 to $60 \mathrm{sec}$.
In contrast, the massed-trials procedure revealed no statistically reliable evidence of a reduction in the intertrial agreement effect as ITI was increased across the same range.

The insensitivity of the intertrial agreement effect to variations in ITI in the massed-trials procedure has led some to suggest that the intertrial agreement effect reflects the influence of a permanent memory carried over from one trial to the next (Edhouse \& White, 1988b; Roitblat, 1984, 1993). However, data from Experiments 1-3 suggest an alternative account. In particular, notice that the magnitude of the intertrial agreement effect is approximately equivalent in the clustered-dyads and massed-trials procedures when the ITI is $60 \mathrm{sec}$ (e.g., compare the bottom graphs in Figure 1, clustered-dyads procedure, and Figure 2, massed-trials procedure). This, of course, is expected because the two procedures were identical when the ITI was $60 \mathrm{sec}$ (recall that the IDI in the clustered-dyads procedure was $60 \mathrm{sec}$ ). Notice, however, that the magnitude of the intertrial agreement effect is considerably larger at the 0 -sec ITI in the clustereddyads procedure than in the massed-trials procedure (e.g., compare the top graphs in Figure 1, clustereddyads procedure, and Figure 2 , massed-trials procedure).

It would appear, therefore, that the difference in the magnitude of the intertrial agreement effect reflects a masking, and hence an underestimation, of the magnitude of that effect at short ITIs in the massed-trials procedure. Two mechanisms that might be responsible for this masking effect in the massed-trials procedure can be suggested. First, the deficit in sample processing that is apparent when short ITIs are employed (Edhouse \& White, 1988b; Roberts, 1980; Roitblat, 1984, 1993; Roitblat \& Scopatz, 1983) tends to depress accuracy toward chance at longer delays. Hence, a floor artifact may result in underestimation of the magnitude of the intertrial agreement effect at short ITIs. Second, when ITIs are short, performance on any particular trial may be influenced by memories carried over from more than one previous trial. If so, the prior memory that intrudes on the current trial may contain information compatible with correct responding on the current trial, even though the sample on the immediately preceding trial is opposite that on the current trial. Similarly, the prior memory that intrudes on the current trial may contain information incompatible with correct responding on the current trial, even though the sample on the immediately preceding trial is the same as that on the current trial. Hence, the functional difference between identical and opposite dyads in the massed-trials procedure would be reduced to the extent that memories from trials earlier than $n-1$ occasionally control performance on Trial $n$.

On this account, the insensitivity of the intertrial agreement effect to variations in ITI in the massed-trials procedure does not reflect the influence of a permanent memory carried over from one trial to the next. Rather, the insensitivity may merely reflect a masking of the true magnitude of the intertrial agreement effect at short ITIs. 
The underestimation of the magnitude of the intertrial agreement when trials are closely spaced may result from a deficit in sample processing and the carryover of memory from several preceding trials.

In summary, the present experiments confirmed a difference between the clustered-dyads and massed-trials procedures with regard to the sensitivity of the intertrial agreement effect to ITI duration. Specifically, such sensitivity is readily apparent in the clustered-dyads procedure, but not in the massed-trials procedure. This phenomenon likely results from the operation of floor effects and the carryover of memory from multiple prior trials in the massed-trials procedure that mask the true magnitude of the intertrial agreement effect at very short ITIs. These considerations suggest that if one is interested specifically in the carryover of memory between trials, the clustered-dyads procedure is the more appropriate procedure because sample-processing deficits are minimized and memory on the current trial is likely to be influenced only by memory on the immediately preceding trial.

\section{REFERENCES}

COHEN, J. S., \& ARMSTRONG, D. L. (1996). Sources of intertrial proactive interference in rats' short-term memory in a delayed successive matching-to-sample modality discrimination. Learning \& Motivation, 27, 485-499.

EdHouse, W. V., \& White, K. G. (1988a). Cumulative proactive interference in animal memory. Animal Learning \& Behavior, 16, 461-467.

EDHOUSE, W. V., \& WhITE, K. G. (1988b). Sources of proactive interference in animal memory. Journal of Experimental Psychology: Animal Behavior Processes, 14, 56-70.

Grant, D. S. (1975). Proactive interference in pigeon short-term memory. Journal of Experimental Psychology: Animal Behavior Processes, 1, 207-220.

Hogan, D. E., Edwards, C. A., \& Zentall, T. R. (1981). Delayed matching in the pigeon: Interference produced by the prior delayed matching trial. Animal Learning \& Behavior, 9, 395-400.

KIRK, R. E. (1968). Experimental design: Procedures for the behavioral sciences. Belmont, CA: Brooks/Cole.

ROBERTS, W. A. (1980). Distribution of trials and intertrial retention in delayed matching to sample with pigeons. Journal of Experimental Psychology: Animal Behavior Processes, 6, 217-237.

RoITBLAT, H. L. (1984). Representations in pigeon working memory. In H. L. Roitblat, T. G. Bever, \& H. S. Terrace (Eds.), Animal cognition (pp. 79-97). Hillsdale, NJ: Erlbaum.

RoITBLAT, H. L. (1993). Representations and processes in working memory. In T. R. Zentall (Ed.), Animal cognition: A tribute to Donald A. Riley (pp. 175-192). Hillsdale, NJ: Erlbaum.

RoItBlat, H. L., \& ScopaTz, R. A. (1983). Sequential effects in pigeon delayed matching-to-sample performance. Journal of Experimental Psychology: Animal Behavior Processes, 9, 202-221.

(Manuscript received April 3, 2000; revision accepted for publication June 12,2000.) 